\title{
Measurement Techniques for Identifying Polarity Dependence of Ion Injection in Transformer Oil
}

\author{
Joachim Schiessling ${ }^{1}$, Deepthi K. Ramesh ${ }^{1}$, Yuriy V. Serdyuk ${ }^{2}$ and Olof Hjortstam ${ }^{1}$ \\ ${ }^{1}$ Power Technologies, ABB Corporate Research, \\ SE-721 78, Västerås, Sweden \\ ${ }^{2}$ High Voltage Engineering, Chalmers University of Technology, \\ SE-41296 Gothenburg, Sweden
}

\begin{abstract}
Charge injection from metallic surfaces into mineral oil is investigated utilizing transient currents recorded in coaxial cylindrical electrode systems with different degrees of inhomogeneity of electric fields. The currents obtained with single polarity and reverse polarity methods demonstrate differences in their behavior depending on the geometry and type of the test voltage. The data show clear relation between the sign of the current and the polarity of the applied voltage, which is attributed to the polarity dependence of the ion injection from oil-metal interfaces. The results of the experiments are discussed in terms of relaxation time of the system and time of flight (drift time) of ions in the oil gap.
\end{abstract}

\section{Introduction}

HVDC power transmissions are beneficial for transporting electrical energy from remote generation sites to consumers over large distances with minimal losses. A key element of this technology is a converter transformer which links ac and dc parts of a power grid. The electrical insulation system of such a transformer is exposed to both kinds of stresses and should also be able to operate properly during transients (e.g., dc polarity reversal) and to withstand lightning and switching over-voltages. The insulation is usually built utilizing solid (paper, press-board) elements immersed in mineral transformer oil. Under strong dc electric fields acting inside the insulation system, electrical charges can be injected from dielectric and metallic surfaces into oil and give rise to space charge accumulation. This is undesirable, but practically unavoidable inherent phenomenon in hvdc insulation and it needs to be deeply understood in order to develop proper design rules for converter transformer insulation. In the present paper, the applicability of a single polarity and reversed polarity methods [1,2] for characterizing ions injection from metallic surfaces into transformer oil is discussed. Polarity dependence of injection is analyzed based on the results of the measurements of the currents through oil samples in response to the applied test voltage.

\section{Experimental set-up and procedure}

Two types of cylindrical coaxial test cells providing different electric fields in oil layer were utilized. In the experiments, the test cell was filled with transformer oil (Nynäs Nitro 10XN) and it was connected to an electrometer (Keithley 6517A) which was used as both a test voltage source and a current meter. The electrometer was connected to a PC, where measured data were collected and processed. Before test voltage application, the cells were grounded to ensure relaxation of space charges and to avoid electrostatic charging. During the measurements, the outer electrode was grounded while the inner cylinder was energized.

Different test conditions were realized in the two test cells by utilizing electrodes of different dimensions as shown in Figure 1. Thus, cell A was a commercially available stainless steel cell from IR-lab [3], where the difference between the radiuses of the inner $(21.5 \mathrm{~mm})$ and outer electrodes $(25.1 \mathrm{~mm})$ was rather small, thus the field in the gap $d=3.6 \mathrm{~mm}$ was almost homogenous. For this cell, the magnitude of the applied test voltage was $U=400 \mathrm{~V}$. With typical ionic mobility $\mu \sim 10^{-9} \mathrm{~m}^{2} / \mathrm{Vs}$ [4] and assuming uniform field distribution in the oil gap, the transit time for ions can be evaluated as $\tau_{\text {tran }}=d^{2} / \mu|U| \sim 30 s$ [5]. Test cell B was custom made with polished stainless steel electrodes. The radiuses of the inner and outer electrodes were $12.5 \mathrm{~mm}$ and $47.5 \mathrm{~mm}$, respectively, providing oil gap of $35.0 \mathrm{~mm}$. With the test voltage of $1000 \mathrm{~V}$, this yielded an estimate for the transit time $\sim 1400 \mathrm{~s}$. Note that the electric field in oil gap in cell B was non-uniform due to the large ratio of the radiuses.

(a) Cell $\mathrm{A}$

(b) Cell B

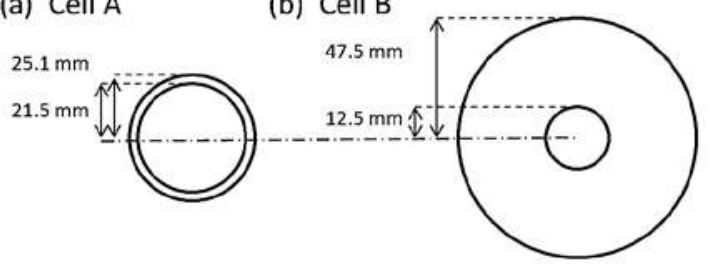

Figure 1 Dimensions of the measurement cells used. 
Before filling the cells with oil, they were thoroughly cleaned and dried. The resistivity of the oil was controlled prior and after the current measurements. For this, the frequency response at low voltages [4] was utilized and the equilibrium resistivity $\rho \sim 3 \cdot 10^{13} \Omega \mathrm{m}$ was obtained. With this value and typical dielectric constant of oil $\varepsilon_{r}=2.3$, the estimate for the dielectric relaxation time of the system is $\tau_{r e l}=\varepsilon_{0} \varepsilon_{r} \rho \sim 600 \mathrm{~s}$. Note that the ration between the transient and relaxation times $k=\tau_{\text {tran }} / \tau_{\text {rel }}$ characterizes dynamic properties of the systems and can be utilized for interpretation of the results of the measurements.

\section{Results}

\subsection{Single polarity method}

The transient currents measured in the test cells A and B after application of the test voltage $(400 \mathrm{~V}$ and $1000 \mathrm{~V}$, respectively) are shown in Figure 2 for both polarities. Note that for comparison purposes, the absolute values of the currents are presented. As one can observe, high capacitive current appeared on all the recorded traces immediately after voltage application and it was followed by a fast decay. It can be noticed that even after several hours measurement, the data still showed a slope in the current (see insets) indicating long-lasting relaxation processes, which, however, are not investigated further in the present study.

The polarity effect is clearly seen in the current traces at a few tens of seconds after the test voltage application.

(a)

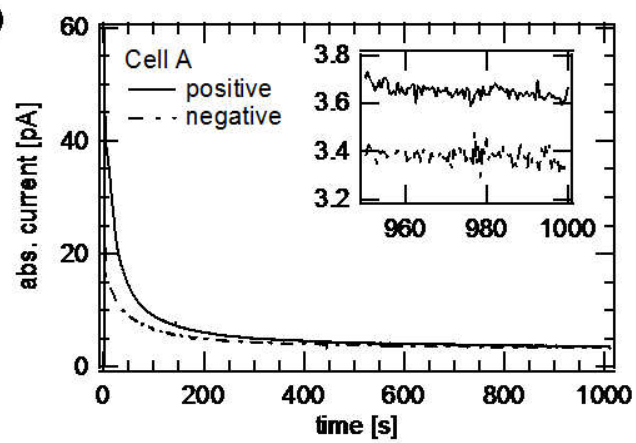

(b)

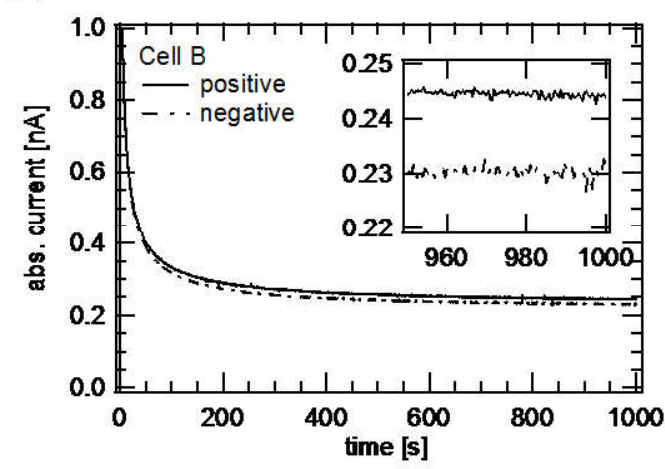

Figure 2 Result from single polarity measurements. Insets show a close-up of the current at longer times.
The positive current is in general higher that the negative one and this trend is observed in the data obtained with the both cells. Note that the currents measured in the test cell B (Figure $2 \mathrm{~b}$ ) are much higher than those in the cell A due to much stronger electric fields in oil and, in particular, at the surface of the injecting (inner) electrode. However, the polarity effect is more pronounced in the more homogeneous field provided by test cell A.

\subsection{Reverse polarity method}

The adopted test procedure consisted of three steps: first, the test voltage was applied during time $t_{0}$; further, the polarity was reversed for $t_{0}$; and, finally, the polarity was switched back. The currents measured in cells A and B on the last step are shown in Figure 3.

The current traces recorded in cell A (Figure 3a), which provided almost homogeneous field, show peaks corresponding to the time scale of the ion transit time due to ion-drift phenomena, which occurred in the small oil layer when applying the polarity reversal method. The height of the peaks is largest at negative polarity. After the peak, the current decays with time and the magnitude of the positive current is lower than that of the negative one. In contrast, the currents measured in

(a)

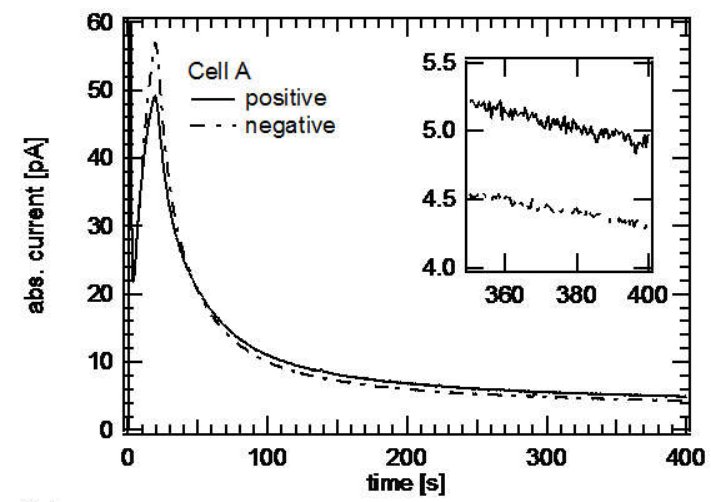

(b)

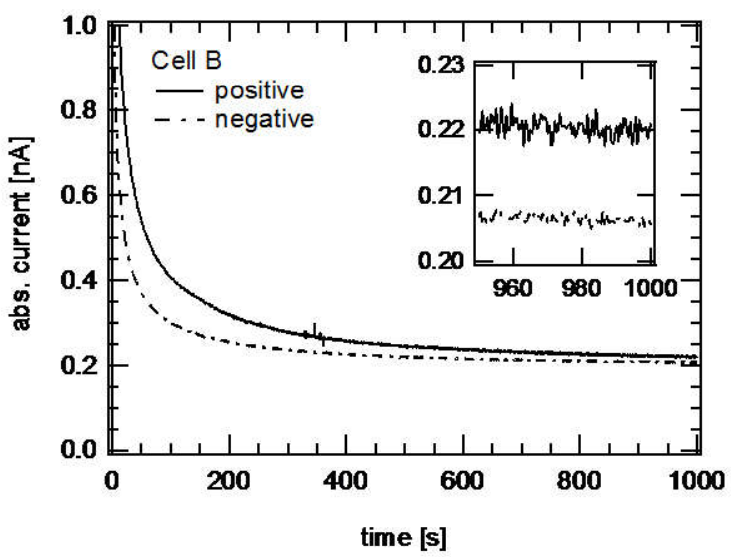

Figure 3 The currents measured with reverse polarity method. The voltage was reversed after $\mathrm{t}_{0}=600 \mathrm{~s}$ and $1000 \mathrm{~s}$ for cell A and B, respectively. The insets show the current at longer times. 
cell B decay more rapidly at both polarities, Figure $3 b$, and the positive current is in general higher than the negative one, similarly to the single polarity results.

\section{Discussion}

In general, all the measurements show that the current decreases with time after test voltage application. At the beginning of the decay, a high slope is observed and the current becomes almost constant at longer times. The qualitative and quantitative differences in the currents measured in different test cells and using different methods can be attributed to behavior of ions injected from oil-metal interfaces

Thus, there is a principle difference in distributions of ions within oil layer when the test voltage of single polarity or reversed polarity is applied. Prior to voltage application, the oil is in its equilibrium state having the corresponding equilibrium concentration of ions. After application of the test voltage, a sweep out phenomenon takes place and the charge carriers from the volume of oil drift in the field towards the electrodes thus reducing ions concentration in the bulk. This process gives rise to the large transient displacement current as seen in the currents recorded for both cells, Figure 2. The majority of ions pile-up at the electrodes as space-charges, which in turn reduce the electric field in the gap until the system reaches a steady-state. This may take extremely long time and, as it is shown in the inset in Figure 2, a small slope in the current trace can be observed even after several hours.

The initial conditions in reversing polarity measurements are different and the swept-out of ions from the bulk may already produce space charge layers at the electrodes. When polarity reversal takes place, the space charges will move to the counter electrodes and their movement gives rise for the peak in the current on time scale of several seconds [6]. For cell A with its thin oil gap, this is observed and shown in Figure 3a. For cell $\mathrm{B}$, the thickness of the oil gap is larger and the ions are able to recombine while crossing the gap and, therefore, no distinct peaks can be seen in the data, Figure $3 b$.

As it was mentioned above, significantly higher steadystate currents were measured when positive voltages were applied to the inner electrodes of the test cells. The steady-state currents estimated from the data presented in Figures 2 and 3 are summarized in Table 1. The observed polarity dependence appear due to the inhomogeneous electric fields in the test vessels. Thus in cell $\mathrm{B}$, the electric field is stronger at the inner electrode than in cell A. Therefore, the injection of charges (ions) of the same polarity as the potential applied to the inner electrode is enhanced with respect to the outer electrode. This, however, is different in the case of reversed polarity. The higher magnitude of the negative current observed in Figure $3 \mathrm{a}$ for cell $\mathrm{A}$ at times $\sim 20$ s may occur due positive space charges accumulated in the oil at the high field electrode prior to polarity reversal. When the potential applied to the inner
Table 1 Currents from Figures (1) and (2) at long times.

\begin{tabular}{|c|r|c|c|}
\hline \multicolumn{2}{|c|}{ currents } & $\begin{array}{c}\text { Cell A } \\
{[\mathrm{pA}]}\end{array}$ & $\begin{array}{c}\text { Cell B } \\
{[\mathrm{nA}]}\end{array}$ \\
\hline single & positive & 3.62 & 0.244 \\
polarity & negative & 3.37 & 0.231 \\
\hline reverse & positive & 4.97 & 0.220 \\
polarity & negative & 4.31 & 0.206 \\
\hline
\end{tabular}

cylinder changes its sign, the ions present in its close vicinity move rapidly to the electrode providing relatively high current. Further consideration of the polarity and field dependent injection of oil ions from metallic surfaces is provided in [7], where results of computer simulations are compared with measured data obtained with Kerr electro-optic method.

\section{Conclusions}

Measurements of ionic currents in oil have been carried out using single and reverse polarity methods with the test cells providing different degrees og inhomogeneity of the electric fields. In all cases, the measurements yielded significantly stronger positive currents on the time scale longer than the transit time of ions through the oil gap. The reversed polarity measurements performed in the smaller gap show enhanced current peak at negative polarity, that is believed to be associated with injection of positive space charges during the cycle prior the measurement. Based on the obtained results from both the methods, it can be confirmed that the ion injection from metal-oil interface is stronger for positive polarity.

\section{References}

[1] L. Yang, M. Shafiee Zadeh, J. Schiessling, O. Hjortstam, Y. V. Serdyuk, and S. M. Gubanski, "Measurement of Ion Mobility in Transformer Oils for HVDC Applications", Proc. Int. Conf. High Volt. Eng. Appl., Shanghai, China, Sept. 2012, p. 464.

[2] V. Novotny and M. A. Hopper, "Transient Conduction of Weakly Dissociating Species in Dielectric Fluids", J. Electrochem. Soc., Vol. 126, pp. 925

[3] IR-labs, France

[4] L. Yang, S. M. Gubanski, Y. V. Serdyuk, and J. Schiessling "Dielectric Properties of Transformer Oils for HVDC Applilcations" IEEE-TDEI, Vol. 19, No. 6, 2012, p. 1926

[5] U. Gäfvert, A. Jaksts, C. Törnkvist and L. Walfridsson, "Electric Field Distribution in Transformer Oil", IEEE TEI, Vol. 27, No. 3, 1992, p. 647

[6] O. Hjortstam, J. Schiessling, Y. V. Serdyuk and S. M. Gubanski, "Measurements of Ion Mobility in Transformer Oil: Evaluation in Terms of Ion Drift", Proc. CEIDP 2012, Montreal, Canada, Oct. 14-17, p.496

[7] O.Hjortstam, C. Sonehag and J. Schiessling "Comparison of Simulated and Measured Field Dependent Charge Injection in Mineral Oil under dc Bias", NORDIS Conf. Proceeding, Trondheim, Norway, June 2013. 